\title{
OBSERVATIONS OF THE HORIZONTALLY ORIENTED CRYSTALLINE PARTICLES WITH A SCANNING POLARIZATION LIDAR
}

\author{
Grigorii Kokhanenko*, Yurii Balin, Anatolii Borovoi, Marina Klemasheva, Sergei Nasonov, \\ Mikhail Novoselov, Ioganes Penner, Svetlana Samoilova \\ Institute of Atmospheric Optics, Siberian Branch of Russian Academy of Sciences, Tomsk, Russia \\ *Email:kokh@iao.ru
}

\begin{abstract}
Scanning lidar LOSA-M3 makes it possible to measure the polarization characteristics of backscattering signals from aerosol and clouds at wavelength 532 and $1064 \mathrm{~nm}$. The lidar transceiver is placed on a scanning column, which allows changing the direction of sounding within the upper hemisphere at a speed of 1 degree per second. The polarization characteristics of the transmitter (linear or circular polarization) can be changed by rotating the phase plates synchronously with the laser pulses. Conical scanning of the lidar allows identifying cloud areas with preferential zenith or azimuthal orientation of the crystal particles. The article presents the results of observations of the cloud polarization structure carried out in Tomsk. Methods of the calibrations of lidar polarization channels are described.
\end{abstract}

\section{INTRODUCTION}

Cirrus clouds cover a significant part of the earth's surface. They have a significant impact on the radiation balance and climate. Radiation reflection by and transmission through these clouds depends essentially on particle shapes and orientations ${ }^{1-3}$. The use of the scalar model, even assuming a random orientation of the particles, gives a noticeable error in the calculation of the radiation passage ${ }^{4}$. Traditionally, most radiative models of cirrus suppose a random orientation of crystals ${ }^{5}$. According to Ref. 6, in the presence of a preferential orientation of cloud particles, lidar depolarization measurements can yield ambiguous results. Numerous work on sounding cirrus clouds by polarization lidars $^{6-10}$ have shown that ice crystals in cirrus clouds often show not only a predominant orientation relative to the horizon (zenith orientation), but they also have a predominant orientation in the horizontal plane (azimuthal orientation) connected with the direction of the wind. The lidar polarization measurements for which the lidar was directed or strictly vertical, or deviated from the vertical direction by some fixed angle, ${ }^{11-13}$ made it difficult to interpret the obtained data. The advantage of scanning lidars is the ability to diagnose the preferential orientation of crystals in the clouds. Two wavelengths of the radiation source of $532 \mathrm{~nm}$ and $1064 \mathrm{~nm}$ provide additional information on the microphysical parameters of the clouds

\section{METHODOLOGY}

\subsection{The lidar system}

Lidar LOZA-M3 is a continuation of a series of small-sized lidars LOZA-MS and LOZA$\mathrm{M} 2^{14,15}$, developed and operated in the IAO SB RAS. All these lidars are intended primarily for use in expeditionary conditions, which imposes certain restrictions on the weight-dimensional characteristics and the energy potential of the device. The main features of LOZA-M3 lidar are: 1) - automatic scanning drive, which allows to change the direction of sounding within the upper hemisphere at a speed of up to 1 degree per second with the accuracy of the set angle measurement no worse than 1 minute, and 2) polarization channel that allows to determine some elements of the scattering matrix of aerosol particles at two wavelengths simultaneously.

The optical scheme of the lidar is shown in Fig. 1. The Q-Smart 850 (Quantel) laser 1 with a fundamental harmonic energy of $850 \mathrm{~mJ}$ is used, the repetition frequency is $10 \mathrm{~Hz}$. The radiation is linearly polarized, but the plane of polarization of the second harmonic $(532 \mathrm{~nm})$ is rotated by $45^{\circ}$ relative to the first. To reconcile the polarization planes, a phase plate 2 having a phase shift of 20 wavelengths for $\lambda=532 \mathrm{~nm}$ and 9.5 wavelengths for $\lambda=1064 \mathrm{~nm}$ is used. Turning the plate achieves the coincidence of the planes of polarization for two harmonics. The Glan prism 3 improves the polarization contrast of the radiation. The phase plate 4 , optionally a quarter wave $\lambda / 4$ or half-wave $\lambda / 2$, serves to rotate the plane of polarization of the radiation $(\lambda / 2)$ or to transform the polarization 
state $(\lambda / 4$, linear-circular $)$.

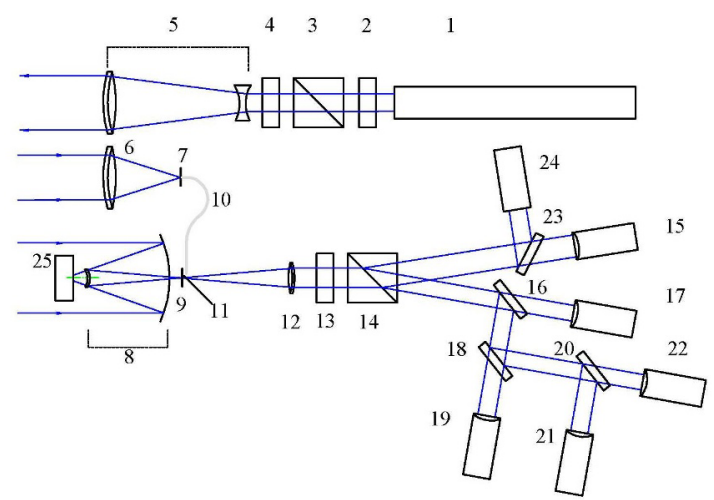

Fig. 1. Optical circuit of the lidar LOZA-M3

The Wollaston prism 14 divides the beam into components with orthogonal polarization, which are further divided along the wavelengths by dichroic beamsplitters. Unlike schemes in which wavelength division is carried out before the separation of polarization components, there is no distortion of the polarization state when reflected from dichroic elements and there is no need to apply laborious calculations of the instrument vector and correction of the measured polarization ${ }^{16}$.

\subsection{Calibration of the polarization channels}

To measure the depolarization ratio of the backscattered radiation, the relative sensitivity of detectors must be known. We use the technique of continuous rotation of $\lambda / 2$ phase plate (13 at Fig. 1), installed in receiver module. Let us assume that laser radiation is linearly polarized (the initial vector is $\mathbf{S}_{0}^{L}=[1,1,0,0]^{T}$ ). If we set the $\lambda / 2$ phase plate before the polarization prism with matrix $\mathbf{L}(\varphi), \varphi$ is the rotation angle, the scattered Stokes vector will

be $\mathbf{S}=\mathbf{L M S}_{0}^{L}=\left(\begin{array}{c}m_{11}+m_{12} \\ \left(m_{12}+m_{22}\right) C_{4}-\left(m_{13}+m_{23}\right) S_{4} \\ \left(m_{12}+m_{22}\right) S_{4}+\left(m_{13}+m_{23}\right) C_{4} \\ m_{14}+m_{24}\end{array}\right)$,

where $C_{4}=\cos 4 \varphi, S_{4}=\sin 4 \varphi$. Polarization components $I_{\|}, I_{\perp}$ are different, but the integral over angle $\varphi$ : $\int_{2 \pi} I_{\|, \perp} d \varphi=\pi\left(m_{11}+m_{12}\right)$ is the same for each polarization component regardless of the values of matrix elements. Using the continuous rotation of the $\lambda / 2$ phase plate, we can measure the relative sensitivity of the detectors and calibrate polarization channels.

\section{RESULTS}

\subsection{Zenith scanning at $1064 \mathrm{~nm}$ wavelength}

Below we present the results of the crystal cloud observations at the zenith scanning. Scanning was performed at a rate of 0.5 degrees per second. The angle is measured from the vertical position of the radiation beam, which was set with an error of \pm 5 minutes. In this case, the scan was carried out with the

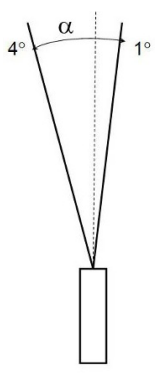
lidar axis passing through the zenith (ranging from $-1^{\circ}$ to $+4^{\circ}$ ), which allowed controlling the accuracy of the lidar vertical setting.

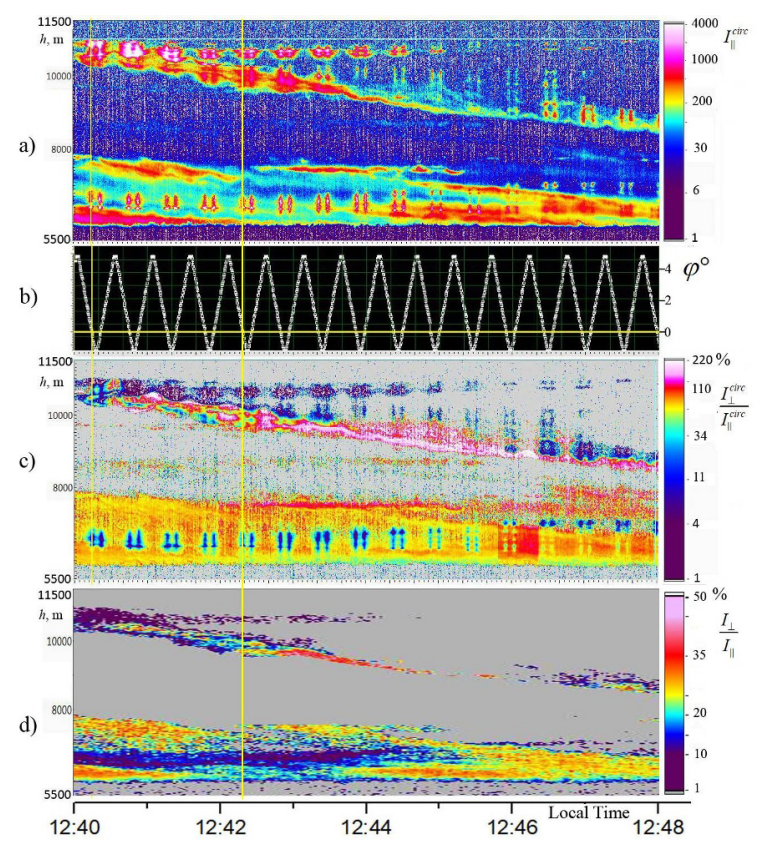

Fig. 2. Cloud structure, observed with zenith scanning

Figure 2 shows the lidar data for 01.10.2018. The tilte angle is shown in Fig.2.b. Upper frame (2.a) indicates the parallel component $I_{\|}^{\text {circ }}$ (circular initial polarization) in ADC codes (04096). Fig. 2c shows the depolarization ratio $\delta=I_{\perp} / I_{\|}$. Positions of vertical orientation $\alpha=0$ (one of them is indicated with yellow line) are characterized by maximal intensity $I_{\|}$and minimal $\delta$. The bottom frame $2 \mathrm{~d}$ (depolarization ratio for linear polarization) shows the record from lidar LOSA-S, placed near the LOSA-M3 lidar and permanently oriented vertically.

Fig. 3 indicates the dependence of the signal intensity on the tilt angle $\alpha$, obtained in measurements on 02.06.2018. The abscissa shows the tilte angle $\alpha$, ordinate shows the intensity in ADC codes. The record in Fig. 3a was registered at $12^{00} \mathrm{LST}$ with averaging of the characteristics over the layer from $11470 \mathrm{~m}$ to 
$11600 \mathrm{~m}$. The record $3 \mathrm{~b}$ was made at $10^{00} \mathrm{LST}$ with averaging of the characteristics over the layer from $10980 \mathrm{~m}$ to $11270 \mathrm{~m}$. These records show the opposite cases of the degree of the specular reflection. For 3 a depolarization ratio $\delta=I_{\perp} / I_{\|} \quad \delta=0.006$ at vertical position $(\alpha=0)$ and $\delta=0.11$ at $\alpha=4^{\circ}$. For $3 b \delta(0)=0.24$ and $\delta\left(4^{\circ}\right)=1.42$.
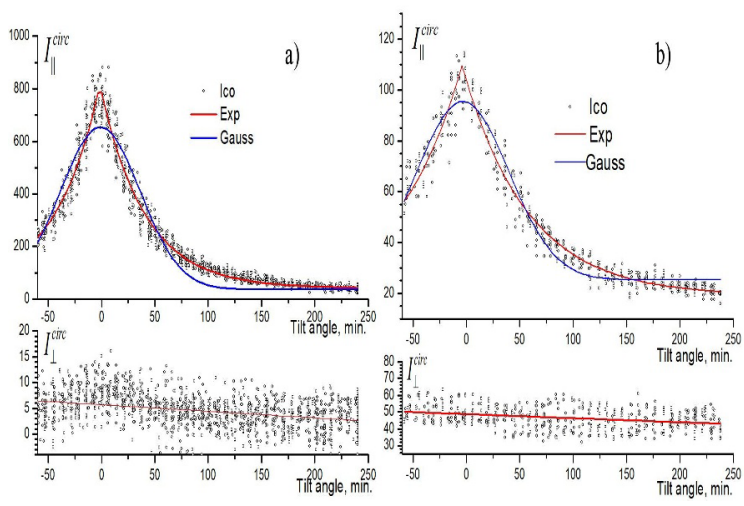

Fig. 3. Dependence of the signal intensity on the tilt angle

All data measured on June 02, 2018 well described by the exponential distribution

$$
I(\alpha)=I_{0}+A \exp \left(-\left|\alpha-\alpha_{0}\right| / w\right),
$$

(red line in Fig. 3), where $w$ determines the width of the distribution, and $\alpha_{0}$ indicates the error of the lidar targeting to vertical. For Fig. 3 a $w=42^{\prime}$, for Fig. $3 \mathrm{~b} w=82^{\prime}$. The shift of the curve maximum is less than 2 minutes, which indicates a good orientation of the lidar to the zenith.

As opposed to Ref. 10 the Gaussian function poorly describes the observed distribution (blue line in Fig. 3). The main difference is determined by the underestimation of the sharp peak at $\alpha=0^{\circ}$. The values $I_{\perp}$ in most cases shows a weak decline with the angle, but its change are smaller than the measurement errors. We can conclude that $I_{\perp}$ are practically independent of the inclination angle. It should be noted that the intensities $I_{\perp}$ for linear polarization in the mirror reflecting zone at some cases do not exceed the noise level of the receiver and are difficult to process. That's why we present here the date of measurements with circular polarization.
Selected cases of observed on June 02 distributions are shown in Fig. 4. These cases cover the entire range of observed widths $w$ of the distribution $I_{\|}(\alpha)$.

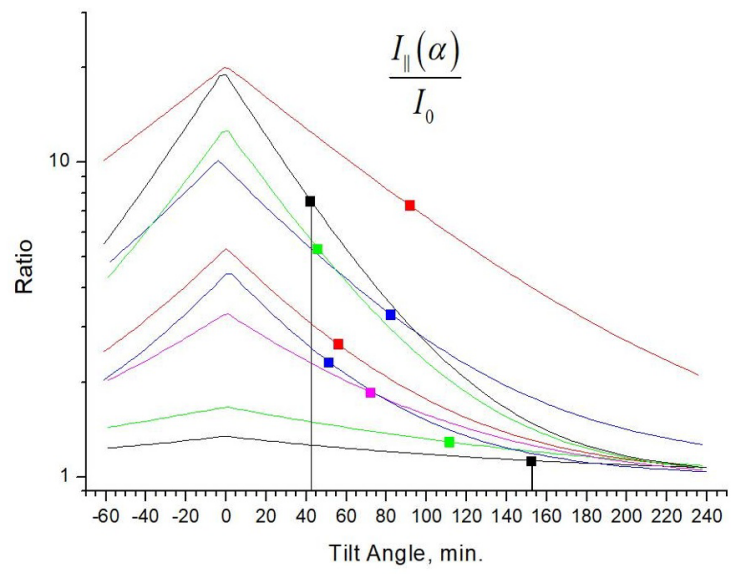

Fig. 4. Different cases of observed distributions $I(\alpha)$

The value of intensity is normalized to the value $I_{0}$ (Eq. 2). If we assume that the signal intensity for $\alpha>>4^{\circ}$ is determined by randomly oriented particles, this value represents the contribution of specularly oriented particles to the total backscattering intensity. We can assume that this corresponds to the distribution of the inclination angles of crystalline particles relative to the horizontal plane.

\subsection{Green and infrared dependencies}

Simultaneous recording of signals allows comparing the angular dependences for wavelengths 532 and $1064 \mathrm{~nm}$. Above, we assumed that the angular dependence of the specular component reflects the angular distribution of particles. But the relative intensity of this component may differ for two wavelength because i) backscattering coefficient for $\alpha>>4^{\circ}$ as rule is different, and ii) the intensity of specular reflection may be differ because of the contour diffraction. Unfortunately, we have not an absolute calibration of recorders for two wavelength, so in the Fig. 5 we present the relative angle dependencies. 


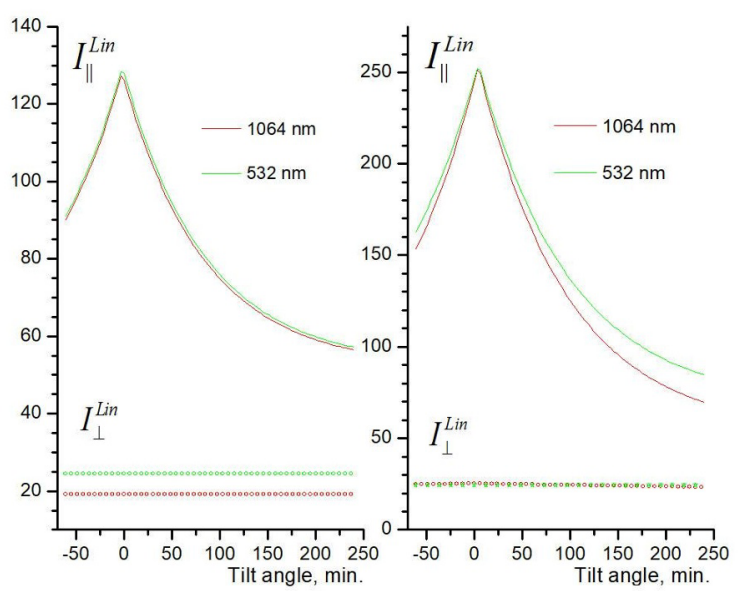

Fig. 5. Angle dependencies of polarization components for $532 \mathrm{~nm}$ (green) and $1064 \mathrm{~nm}$ (red)

The curves are aligned so that the intensity maxima at $\alpha=0^{\circ}$ are equal. We show two cases with maximum angular differences. In the left frame the angle distributions are the same, in the right frame the distribution for $532 \mathrm{~nm}$ is wider $\left(I_{532}\left(4^{\circ}\right) / I_{1064}\left(4^{\circ}\right)=1.5\right)$. In all other cases the distribution width $w$ for $532 \mathrm{~nm}$ is equal or more then for $1064 \mathrm{~nm}$.

\section{ACKNOWLEDGEMENTS}

The work was supported in part by Ministry of Science of Education of RF (Agreement No. 14.616.21.0104, unique identifier RFMEFI61618X0104).

\section{REFERENCES}

[1] Liou, K. N., "Influence of cirrus clouds on weather and climate processes: a global perspective," J. Geophys. Res. 103, 1799-1805 (1986).

[2] Sassen, K., Griffin, M. K., and Dodd, G. C., "Optical scattering and microphysical properties of subvisual cirrus clouds, and climatic implications," J. Appl. Meteorol. 28, 91-98 (1989).

[3] Masuda, K., and Ishimoto, H., "Influence of particle orientation on retrieving cirrus cloud properties by use of total and polarized reflectances from satellite measurements," J. Quant. Spectrosc. Radiat. Transfer 85, 183-193 (2004).

[4] Lawless, R., Xie, Yu, Yang, P., Kattawar, G. W., Laszlo, I., "Polarization and effective Mueller matrix for multiple scattering of light by nonspherical ice crystals," Opt. Express 14, 6381-6393 (2006).

[5] Takano, Y., Liou, K.-N, and Minnis, P., "The effect of small ice crystals on cirrus infrared radiative properties," J. Atmos. Sci. 49, $1487-$ 1493 (1992).

[6] Kaul, B.V., Samokhvalov, I.V., and Volkov,
S.N., "Investigating of particle orientation in cirrus clouds by measuring backscattering phase matrices with lidar," Appl. Opt. 43, $6620-6628$ (2004).

[7] Balin, Yu. S., Kaul, B. V., Kokhanenko, G. P., and Penner, I. E., "Observations of specular reflective particles and layers in crystal clouds," Opt. Express 19, 6209-6214 (2011).

[9] Borovoi, A., Balin, Y., Kokhanenko, G., Penner, I.; Konoshonkin, A., and Kustova, N., "Layers of quasi-horizontally oriented ice crystals in cirrus clouds observed by a two-wavelength polarization lidar," Opt. Express 22, 2456624573 (2014).

[9] Chepfer, H., Brogniez, G., Goloub, P., Breon, F. M., and Flamant, P. H., "Observations of horizontally oriented ice crystals in cirrus clouds with POLDER-1/ADEOS-1," J. Quant. Spectrosc. Radiat. Transfer 63, 521-543 (1999).

[10] Noel, V., and Sassen, K., "Study of Planar Ice Crystal Orientationsin Ice Clouds from Scanning Polarization Lidar Observations," J. Appl. Meteor. 44, 653-664 (2005).

[11] del Guasta, M., Vallar, E., Riviere, O., Castagnoli, F., Venturi, V., and Morandi, M., "Use of polarimetric lidar for the study of oriented ice plates in clouds," Appl. Opt. 45, 4878-4887 (2006).

[12] Hayman, M., Spuler, S., Morley, B., and Van Andel, J., "Polarization lidar operation for measuring backscatter phase matrices of oriented scatterers," Opt. Express 20,2955329567 (2012).

[13] Veselovskii, I , Goloub, P., Podvin, T., Tanre, D., Ansmann, A., Korenskiy, M., Borovoi, A., Hu, Q., and Whiteman, D. N., "Spectral dependence of backscattering coefficient of mixed phase clouds over West Africa measured with two-wavelength Raman polarization lidar: features attributed to ice-crystals corner reflection," J Quant Spectrosc Radiat Transfer 202, 74-80 (2017).

[14] Bairashin, G.S., Balin, Yu.S., Ershov, A.D., Kokhanenko, G.P., and Penner, I.E., "Lidar "LOZA-MS" for investigation of aerosol fields in troposphere," Optical Engineering, 44(7), 071209-1-071209-7 (2005).

[15] Bairashin, G.S., Balin, Yu.S., Klemasheva, M.G., Kokhanenko, G.P., Penner, I.E., and Samoilova, S.V. "Aerosol-Raman lidar "LOSAM2," Quantum electronics, 41(10), 945-949 (2011).

[16] Di, H.G., Hua, H.B., Cui, Y., Hua, D.X., Li, B., and Song, Y.H., "Correction technology of a polarization lidar with a complex optical system," J. Optical Soc. Amer. A., 33(8), 1488-1494 (2016). 